\title{
Argonne National Laboratory Study of the Transfer of Federal Computational Technology to Manufacturing Industry in the State of Michigan
}

By

Charles J. Mueller

November 1991 


\section{Acknowledgments}

This study benefited from the guidance and support of Dr. Jonathan S. Morrell and Mr. Richard Shackson of the Industrial Technology Institute. Dr. Morrell also provided the information on technology needs of selected industries in the State of Michigan and identified individuals to contact for technology transfer projects ielated to manufacturing issues.

This study also benefitted from discussions and interactions with Mr. Todd Anuskiewicz and Dr. Tom Borton of MERRA. It is expected that these discussions will also lead to collaborations involving Argonne and selected software firms within the State of Michigan.

A final note of acknowledgment is due to Mr. Norm Peterson of the Strategic Planning Office at Argonne. Norm has played instrumental roles in initiating and developing numerous collaborations between Argonne programmatic staff and industrial groups such as ITI and MERRA. 


\section{TABLE OF CONTENTS}

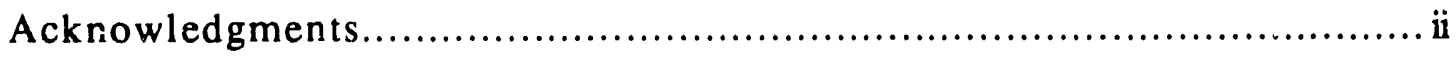

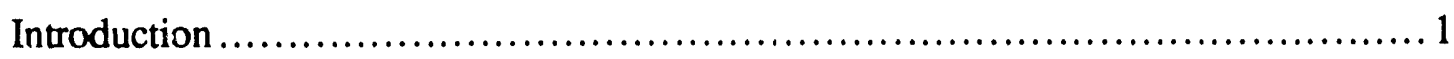

Step 1. Identification Needs....................................... 2

Step 2. Identification of Relevant Technology Areas ....................... 2

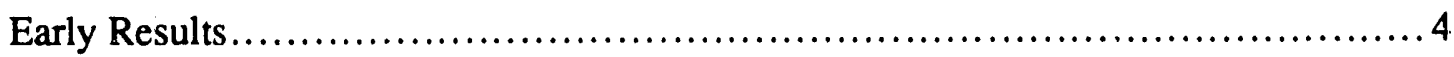

Observations and Recommendations........................................... 5

1. Develop an Effective Industry Needs Statement ............................5

2. Develop Technology Capability Matrix (Federal Laboratories and Universities) . . 5

3. Ensure Mechanisms for Follow-through on Potential Programs ................5

Conclusions and Epilogue................................................ 7

Appendix A Summary of High-Perfornance Computing Capabilities at Argonne

National Laboratory .............................................................. 9 


\section{Introduction}

This report describes a pilot study to develop, initiate the implementation, and document a process to ideritify computational technology capabilities resident within Argonne National Laboratory to small and medium-sized businesses in the State of Michigan. It is a derivative of a program entitled "Technology Applications Development Process for the State of Michigan" undertaken by the Industrial Technology Institute and MERRA under funding from the National Institute of Standards and Technology. The overall objective of the latter program is to develop procedures which can facilitate the discovery and commercialization of new technologies for the benefit of small and medium-sized manufacturing firms. Federal laboratories such as Argonne, along with universities, have been identified by the Industrial Technology Institute as key sources of technology which can be profitably commercialized by the target firms.

Argonne National Laboratory (ANL) is a multi-disciplinary researcin and development institution operated by the University of Chicago for the U.S. Department of Energy. Argonne has an aggressive and innovative technology transfer program that transfers technology in a variety of ways, including cooperative Research and Development, contract research, staff exchange, and licensing of inventions. Argonne's key to successful technological transfer is to seek early industrial involvement and follow through with the adaptation and training needed as new technology moves ultimately to the shop floor. Moreover, Argonne has developed extensive links with industry and with economic development organizations at the state, regional and local levels. These ties, combined with its relationships with universities, make Argonne a major resource for the industrial community.

The process of transferring technology from a federal lab to selected industry groups generally requires the following interdependent steps:

(1) Identification of needs within the industry that can be profitably addressed;

(2) Identification of relevant technology areas and appropriate programs and personnel within the lab;

Identification of potential cooperative projects, contract research projects, or transfer of existing technology that could be brought to bear in the meeting of industry needs;

Development of proposals or contracts to implement the above projects or transfer of technology.

The first two of these steps comprise a prescreening of potential areas of interaction between an industry group such as ITI or MERRA and a national lab or university. Following the screening phase, Step 3 is a project identification phase requiring one-on-one interactions between key technical participants from each organization.

The scope of this project has been limited to developing and documenting the first three steps for the potential application of technology within the broad area of computational science and engineering to areas of importance to small- and met ium-sized firms in manufacturing and manufacturing-related areas. As a result of the work performed in this pilot study, there are ongoing meetings among ITI, MERRA, and Argonne staff to define technology transfer programs in the areas defined in Step 3. The following paragraphs describe the activities undertaken in this pilot study. 


\section{Step 1: Identification of Needs}

Four specific manufacturing or related industry technology areas were initially idenifified by ITI and MERRA for this project: metal stamping, tool and die operations, machine tooling, and plastics. Brief overview descriptions of industry needs in these areas can be found in a report prepared by ITI in 1991 entitled "Technology Needs in Four Sectors of the American Economy: An Analysis in Support of Technology Transfer."

There were eight problem areas identified in this report:

Problem Area \#1: Waste Management

Problem Area \#2; Inspection and Sensing - Batch Processing

Problem Area \#3: Inspection and Sensing -- In Process

Problem Area \#4; Automatic Tool Change

Problem Area \#5: Materials

Problem Area \#6: Thermal Distortion

Problem Area \#7: CAD Data Transfer

Problem Area \#8: CAD Capability

These areas were then scanned by Argonne staff to compare the broad needs of these problem areas in manufacturing with known computational technology areas within the Lab. We then discussed as needed these problem areas with the appropriate program manager within ITI to get further elaboration of industry needs.

\section{Step 2: Identification of Relevant Technology Areas}

The scope of this study limited the investigation of technology areas for technology transfer to that of computational science and engineering featuring high performance computing. This area was chosen as the broad technological capability within Argonne to investigate for technology transfer to Michigan firms for several reasons. First, and most importantly, as a multi-disciplinary Laboratory, Argonne has the full range of scientific and engineering skills needed to utilize leadingedge computing capabilities in many areas of manufacturing.

In support of transferring computational capabilities to various industries, Argonne recently compiled for the State of Illinois a related capabilities matrix for major research and development institutions within Illinois, focusing on computational science and engineering. The institutions included Argonne and Fermilab, the two federally funded national laboratories in Illinois, and eight other universities or computing research centers. The Argonne capabilities matrix, adapted for use in the study herein, is presented as Appendix A. As the matrix shows, Argonne's research programs have substantial components in computational science and engineering, which complement extensive experimental and analytical programs in both the scientific and engineering disciplines, as well as support major initiatives such as the Advanced Photon Source and the Integral Fast Reactor. Since most of the information in Appendix A was developed in the summer of 1991 , it is current and readily adaptable for use in matching Argonne capabilities to various industry sector needs.

A second important reason for choosing computational technology is that the Computing and Telecommunications Division of Argonne has had "hands-on" experience in computing and cornputational technology transfer projects with small to medium-sized, technologically oriented, firms within Illinois. In one particular example, a pilot project was defined to transfer supercomputing technology to Packer Engineering of Naperville, Illinois to model the dynamics of a flexible link and sprocket system for tracked vehicles. Because of the structural and dynamic complexity of link-sprocket systems, ordinary design and analysis methods are costly and timeconsuming on computers available to small firms, but are ideally suited to Cray-class supercomputers. Using the Automatic Dynamic Analysis of Mechanical Systems (ADAMS) threedimensional rigid body dynamics code running on the Cray, Argonne and Packer staff modeled the drive sprocket, idler wheel, and 25 pin-connected track links of a bu!ldozer. 
The results from this analysis have been used to provide a visualization of the dynamic behavior of the track system as well as the numerical data on the displacements, velocities, forces, and loads on each component necessary for designing an improved link sprocket system. Since flexible-link and sprocket transmissions similar to those in the computer model are used by nearly all machines or military that convey rotary power, Packer Engineering hopes to use this analysis capability to build their strength in various markets. Industry or military applications include machine tools, industrial automation, high-speed and power transmissions, and automotive components as well as tracked vehicles per se.

Finally, the Computing and Telecommunications Division has active interdisciplinary, interinstitutional programs currently addressing issues that relate to industrial problems that can be solved using computational science and engineering. An example is a project among staff from Argonne National Laboratory, the University of Illinois at Urbana-Champaign's Center for Supercomputing Research and Development (CSRD), and local firms, both large and small. In this project, the Advanced Software Development and Commercialization Project, CSRD and Argonne scientists are involved in the application of software programs to structural problems of interest to a variety of firms including General Motors and Inland Steel. The application of such software programs to problems affecting physical processes in manufacturing is only a slight extension of this ongoing computational technology transfer project, which it should be pointed out, is funded by the Technology Challenge Grant Program of the State of Illinois. 


\section{Early Results}

A comparison of information in the Technology Needs Report with the Computational Science and Engineering Capabilities Matrix led Argonne investigators to identify several areas for future pursuit with ITI and MERRA program managers. These areas cut across the needs in many of the "Problem Areas" identified earlier (in Step 1). Two areas of computational science identified in the matrix of Appendix A were selected initially by Argonne investigators as obvious areas for further exploration: Material Forming Modeling -- Casting (ANL-38) and Structural Analysis (ANL-44). These computational areas have been the subject of past as well as ongoing collaborations between Argonne and various industrial firms. In the casting area, a consortium has been established to develop a computational capability to model the mold-filling and solidification processes used in typical foundry operations.

In the area of Structural Analysis, various Argonne divisions have a number of projects with industrial organizations to model the response of different structures to dynamic loadings. These run the gamut in size and sophistication, and collectively address in one form or another, all of the identified problem areas. The more complicated include nuclear power safety applications, wherein the reactor vessel and related components are subjected to a number of postulated accident scenarios and their structural response calculated. Interestingly, some of the same nonlinear transient finite element analysis techniques here can be used to model the structural vulnerability (crash-worthiness) of automobile vehicles or components in various impact-contact situations. The computational modeling of components and related materials important to automobile structural vulnerability can readily be seen to be of prime importance not only to the automobile manufacturers, but also to their first and second tier suppliers, many of whom are located in the State of Michigan. These areas are currently being investigated to determine whether collaborative activities between the relevant Argonne programs and selected Michigan firms can be defined.

A third computational technology area identified as an area of strength within Argonne is related to that of Scientific Visualization (ANL-11). In particular the integration of finite element analysis and computer-aided design (CAD) seemed to be a task that would fall under Problem Areas \#7 and \#8 (see Step 1) relating to CAD issues. Fundamentally, CAD and finite element analysis (FEA) deal with the representation of complex geometries using discrete surface and interior points along with a connectivity. While an opportunity to link the technologies, e.g. by creating an FEA input file using a CAD program, is possible and in many cases desirable, it is not always exploited. Many small companies using CAD for product design and conceptualization do not use finite element analysis. Large organizations like Boeing, however, have recognized the value of integrating CAD and FEM. One commercial software package I-DEAS from Structural Dynamics Research Company (SDRC) does provide integration between the two technologies, but at the expense of being restructured for the computational platforms supported by SDRC. In a discussion with Argonne computational scientist Edward Plaskacz, ITI program manager Thomas Phelps observed that the best contribution a national lab could probably make would be to focus on tools for handling the big problems. This is also being investigated as a possible area of collaboration.

A fourth area identified relates to Database Management (ANL-6) but also involves advanced computing methods that cut across a number of high performance computing areas. Creation of databases documenting tool life and operating conditions is an essential cornerstone of so-called factorial studies where all factors believed to influence the outcome of a process are studied from a statistical viewpoint. Statistical neural networks have been used to determine "ideal values" needed for each factor for similar process engineering studies. One such statistical neural network implementation was a study to avoid "thin film" circuit cracking during installation. Process engineers collected statistical data on 17 parameters involved in the fabrication of thin film circuits as imput to a neural networ'.. The network was then trained on the basis of this learning set to accurately predict the number of cracks during fabrication. Once the network had been trained, it was operated in reverse. The number of cracks was input and the network computed values for the parameters that result in minimal cracking of "thin film" circuits during assembly. In a similar manner, neural networks may be constructed relating tool-life to operating parameters. This, of course, cuts across many of the identified problem areas. 


\title{
Observations and Recommendations
}

\begin{abstract}
Although the scope of this study has been limited to investigating a process for applying computational technologies within national labs to manufacturing-related issues important to small and medium-sized firms, we can make a number of generic recommendations which will significantly facilitate the screening phases of effecting technology transfer.
\end{abstract}

\section{Develop an Effective Industry Needs Statement}

An industry group such as Industrial Technology Institute or MERRA representing a large group of firms within an industry sector has a difficult job in effectively identifying and compiling the needs of its constituents in effecting technology transfer. The information provided should be specific enough for technical personnel from the labs, universities, or other outside organizations to determine the relevance of related $R \& D$ activities. On the other hand, a needs statement for an industry sector comprising several hundred or even thousands of relatively small firms cannot identify the specific needs of each of its constituents. Thus, a fair amount of work must be done to collapse the individual needs of participating firms into generic categories amenable to solution from products or spinoffs of activities in $R \& D$ centers. The resulting Needs Statement will have value not only in identifying technology transfer possibilities but in explaining the mission of the industry group to existing or future affiliates and various funding agencies.

The technology needs report cited earlier was useful in providing a general introduction to a rather diverse group of manufacturing-related problem areas. However, it would be very beneficial to have a report that was both more comprehensive and more detailed. While we were able to use the cited needs report to match at a very high level technological areas of Laboratory strength with the identified problem areas, a more refined delineation would facilitate a one-to-one matching of inplace Laboratory programs to much more specifically-defined needs. This would, of course, greatly expedite the subsequent technology transfer steps. From the perspective of an industry group such as ITI or MERRA, it would seem to be very beneficial to have a readily available database that matched the needs or potential needs of affiliated companies to the identified need categories.

\section{Develop Technology Capability Matrix (Federal Laboratories and Universities)}

The expertise in a particular technology is generally widely dispersed in a federal lab and probably even more so in a major university. Unless a matrix of the type used in this report is available, it is likely that potential areas of expertise will be missed in the initial screening of applicable R\&D activities. The matrix provided in Appendix A was the result of a considerable amount of work by the members of the Computing and Telecommunications Division of Argonne. But it also required contributions and careful review from most of twenty odd programmatic divisions at the Laboratory. In short, the compilation of this matrix was a major effort -- but without such a screening, with its concomitant level of detail, the opportunity for missing a relevant R\&D activity is great. Often the technology transfer office within a federal lab has only broad, rather general descriptions and concomitant knowledge of the capabilities within the programmatic divisions available for their use in facilitating technology transfer. In fact, to our knowledge, the matrix used in this project is unique.

\section{Ensure Mechanisms for Follow-through on Potential Programs}

Once potential areas of $\mathrm{R} \& \mathrm{D}$ have been identified for their potential in addressing a selected technology need by an industrial firm or industry group, it is imperative that a mechanism be provided to identify projects that will address the needs. This requires one-on-one interaction. The method that is currently used at Argonne has had considerable success. Appropriate industry 
representatives are invited to hear presentations of Argonne programs selected for potential applicability. Follow-up is then pursued, as appropriate, by the individual laboratory staff and their industry counterpaits. The Technology Transfer Office at Argonne generally plays the role of facilitator by guiding the parties as to the potential contractual vehicles available.

A brief summary of those vehicles through which Argonne works with industry is provided below. The most appropriate vehicle will of course depend on the technical goals of the collaboration as well as the alignment of these goals with DOE's technical mission.

Consortia and Alliances. In addi ion to one-on-one interactions, Argonne works with industry on a group basis and has formed consortia and alliances involving industry and universities in several technical areas. These areas currently include metal casting, advanced ceramics, high-temperature superconductivity, supercomputing, and biotechnology.

Cooperative Research and Development Agreement (CRADA). A CRADA allows federal laboratories to do cost-shared research with private companies and other organizations, including universities. It is a cost-shared agreement under which Argonne and the company contribute personnel, equipment, and facilities to a cooperative effort. The CRADA was created specifically for cooperative $R \& D$, with the interests of the private sector in mind. Key features include the withholding of sensitive data for up to five years.

Cost-shared Contracts/Subcontracts. These contracts for joint work are tailored to meet specific company needs and may involve cash or in-kind arrangements. The effort must be mutually beneficial to the company and the government. Each partner gerierally keeps the intellectual property developed and shares information.

Industry Exchange Programs. Private companies may exchange staff with Argonne for mutually beneficial growth in specific R\&D areas. Industry exchanges are paid by some combination of funds from industry and Argonne. Exchange periods range from six months to one year.

\section{Licensing of Inventions and Copyrighting of Computer Programs . Argonne} inventions and software are available for licensing or copyrights. Argonne's work produces many innovative ideas with commercial potential. As these are reported, they are reviewed for technical feasibility, value to industry, and the more promising are identified for commercialization with preference to U.S. industry.

Research Facilities. Certain research facilities may be accessed by private companies and universities. Facilities are available on a nonproprietary basis, where no fee is charged for projects that meet a set of criteria, or a proprietary basis, where the user is bilied.

Work for Others. Under a sponsor-funded contract, the laboratory performs work for industry. Some restrictions apply: the lab cannot compete with private industry and the effort must fit the lab's mission. Intellectual property rights are generally offered to the sponsor. 


\section{Conclusions and Epilogue}

This study has presumably accomplished two objectives: (1) it has shed light on how technology transfer is or can be more effectively accomplished between ITI, MERRA, and their affiliates and a federally funded research institution like Argonne National Laboratory; (2) it has initiated technology transfer investigations among ITI, MERRA, and Argonne to address some specific manufacturing-related problem areas of concern to State of Michigan industrial firms.

It is alsc hoped, of course, that this report and the interactions that have taken place as a result will spawn technology transfer activities between Argonne and Michigan industry. The matrix provided in Appendix A identifies many areas of computational science and engineering thas extend to areas outside that of the manufacturing industry. A spinoff of this project has been the identification of several technologically-oriented but nonmanufacturing-related firms by MERRA as candidates for collaboration.

Finally, as identified in this report, institutions such as Argonne can bring resources to bear that extend beyond that of computing and computation. Argonne has been given the charge by the Department of Energy to make technology transfer to industry an integral part of its mission. As a Laboratory, it has enthusiastically welcomed this change. 
APPENDIX A

SUMMARY OF HIGH-PERFORMANCE COMPUTING CAPABILITIES

AT

ARGONNE NATIONAL LABORATORY

sis

9

戠 


\section{Matrix}

\section{Argonne High Performance Computing and Communications Capabilities}

\section{Program Components}

High Performance Computing Syateme(HPCS)

Reacurch for Fivure Generation Systems

Evaluation of Early Syatems

Syrtem Design Tools

Advanced Prototype Syatem:

Advanced Softwe Technolvey and Akorthm (ASTA)

Softwere Suppon for Grand Challengu (See "CSeE Applicutions")

Softwere Components and Tools

Compatational Techniques

High Performance Computing Revearch Centex

Nationad Resesch and Education Network(NREN)

Interagency Interim NREN

Bualc Resururch and Human Resources (BRHR)

Basic Research

Rexcarch Participation and Training

Infrastructure

Education, Training and Curriculum

Computing and Telecommunications Technologies

Advanced Computing Syatem

Vector Sipercomputers

Parallel Procensing

Distributed Computing

Compllera/ Programming Syatema

Compiler Performance

Data Communications

Graphics Networks

Teleconferencing

High Speed Nefworking

Data Management and Storage

Datebace Management

Visualkation and Imaping

Image Archiving Systems

Scientific Viaualization

Interactive Viaualization

Distributed Visualization

\section{Computational Science and Engineering Applications}

Blology and Ufre Sclences

Genome Resenrch

Radiation Biology

Structural Biology

Computational Biology

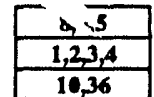

10,36

\section{Chemical Rngineering}

Multi-phase Flow

Slurry(Coal) Systems

\begin{tabular}{|c|}
\hline $4,5,11$ \\
\hline $4,9-12,36$ \\
\hline 4,5 \\
\hline $5,1,2,8$ \\
\hline
\end{tabular}

Chemistry

Fundamental Invextigations

Comburtion Procesuea

Molecular Dynamica/Atomic, Molecular and Electronic Structure

Condensed-Matter Physics

Crystal Growth

\begin{tabular}{|c|}
\hline 17 \\
\hline 18 \\
\hline 19 \\
\hline
\end{tabular}

Energy Syctam:

Systems Analysis and Simulations

Environmentel Sciences

Earth Sciences

Global Climate Modelis:

Atmospheric Sciences

Ucean Circulation Simulation

High Energy Physica

Quantum Chromodynamics

Detector Dexign

Daxn Acquisition and Analysis

Accelerator Design and Simulation

Materials Science

Electronic Structure

Bulk Solids and Surfaces

Superconductivity

Neutron-Scattering Research

Mathematlcs and Numerical Methods

Algorithmic Research

Linear Algebra

Agproximation Techniques

Optimization and Control

Differential Equations

Non-Numerical Methods

Programming Environments and Performance Anal yais

\begin{tabular}{|c|}
\hline$\frac{22}{21,22}$ \\
\hline$\frac{21,22}{22}$ \\
\hline
\end{tabular}

Mechanical Engineering

Heat Transfer

Material Forming Modeling-Casting

Slurry Modeling

Computational Fluid Dynamica/Mechanics

\begin{tabular}{|c|}
\hline$\frac{23}{2425}$ \\
\hline 24 \\
\hline 25 \\
\hline
\end{tabular}

Nuclear Engineering

Reactor Derign

Reactor Safety and Transient Analysis

Fusion Systems

Onlins: Reactor Diagnostics

Structural Engineering

Fatique and Failure Analysis

Earthquake Analysis and Response

Structural Analysis

Crashworthineas

\begin{tabular}{|c|}
\hline 26 \\
\hline 27,26 \\
\hline 23,26 \\
\hline 30 \\
\hline
\end{tabular}

\section{Technalogy Transfer}

Industrial Partners in Computing

Industrial Partners in Science and Engineering

Education, Training and Human Resources

\begin{tabular}{|c|}
\hline 31 \\
\hline$\frac{43,31}{32,31}$ \\
\hline$\frac{33,31}{34,31}$ \\
\hline 35,31 \\
\hline 36,31 \\
\hline
\end{tabular}

\begin{tabular}{|c|}
\hline 37 \\
\hline 38 \\
\hline 39 \\
\hline $37,41,42$ \\
\hline
\end{tabular}

\begin{tabular}{|c|}
\hline $4,37,41$ \\
\hline 41,37 \\
\hline 42 \\
\hline 45 \\
\hline
\end{tabular}

\begin{tabular}{|c|}
\hline 41 \\
\hline 40,41 \\
\hline 4,40 \\
\hline 43,44 \\
\hline
\end{tabular}

\begin{tabular}{|c|}
\hline 48,31 \\
\hline 4631 \\
\hline
\end{tabular}




\title{
Summary of High-Performance Computing Capabilities
}

at

\author{
Argonne National Laboratory
}

The following information complements the matrix at your left in summarizing high-performance computing capabilities and activities at Argonne National Laboratory. In view of current U.S. challenges for more efficient energy sources, environmental and medical improvements, meteorological studies, advancements in industria! designs, and overall national security, no capabilities or activities are more essential to the continued evolution and improvement of our environment than the computational research described below.

AREA

KEY

\section{Program Components}

High Performance

Computing Systems

Research for Future

Generation Systems

Evaluation of Early

Systems
ANL-1 Argonne has joined several leading U.S. research institutions in forming the Concurent Supercomputing Consortium, through which researchers from the geogr aphically separated member institutions have access to the Touchstone DELTA system, currently the world's fastest computer, with a peak speed of 32 gigaflops. Argonne is also a consortial member of the National Science Foundation Science and Technology Center for Research on Parallel Computation. (See also ANL-2.)

ANL-2 The Advanced Computing Research Facility was established in 1984 in recognition of the role that parallel computer architectures will play in the future of scientific computing. It has four principal objectives: (1) to encourage experimentation on computers with innovative designs, (2) to assess the suitability of diverse machines for specific applications, (3) to support Argonne research in parallel computation, and (4) to operate as a national user facility. The ACRF at Argonne currently contains eight commercial multiprocessing systems: a Connection Machine 2 with 16,384 one-bit processors and a total of 128 megabytes of memory, a DAP-520 with 1,024 one-bit processors each having 8 kilobytes of memory, a BBN TC2000 with 45 processors having 192 megabytes of memory, a Sequent Symmetry with 26 pincessors sharing 32 bytes of memory, an Encore Multimax with 20 processors sharing 64 megabytes of memory, an Intel iPSC/860 with 8 nodes (each having 16 megabytes of memory, an Alliant FX/8 with 8 vector processors sharing 64 megabytes of memory, and a Stardent Titan graphics supercomputer with 4 vector processors and 32 megabytes of memory. Linked to one another and to a cluster of more than 70 
workstations, the ACRF computers connect to the Argonne TCP/AP network. ACRF users can access graphics output equipment tape drives, and mass storage devices available through the central Argonne computers. All parallel computers are limited in a single local-area network and are available via local and long-distance telephot.. and natural networks. (See also ANL-1.)

System Design Tools

Advanced Prototypes

Advanced Sortware

Technology and

Algorithms

Software Support for Grand Challenges

Software Comprnents ard Tou's

Computational Techniques

High Performance Computing Research Censers
See ANL-36.

ANL-3 The Argonne Advanced Database and Modeling Research Center researches and develops practical applications of computer technology, focusing primarily on prototype development and technology transfer in such areas as data management, visualization, parallel processing, optical storage, high-speed communications, networking, artinicial intelligence, and expert systems applications, distributed computing, and object-oriented concepts. Thic Center contains a Sequent Symmerry 24-processor plarallel computer, an Alliant VFX/80, two Raster Graphics supercomputers, and a network of approximately 60 Sun workstations, NeXT workstations, IBM-type personal computers, Apple Macintosh computers, and windowing terminals. (See also $A N_{L}-1$.)

ANL-4

Argonne scientists are achieving major computational advances in selected scientific and engineering disciplines by developing algorithms and adapting codes to exploit current and next-generation supercomputers maximally. Special emphasis is placed on multiprocessor architectures with gigaflop and emerging teraflop capabilities with the number of processors ranging to tens of thousands. Emphasis is also placed on implementation of code on distributed platforms such as a connected array of workstations. Argonne scientists are initiating, developing, and performing multidisciplinary collaborative programs in advanced computational science areas with research counterparts in industry and major universities. (See also ANL 5 and ANL-11.)

See ANL-4, 9, 11, 12, and 36 .

See ANL-4 and 5.

ANL-5 Argonne pursues advanced computational science applications-oriented research by (1) initiating collaborative software development programs for adaptation of production and state-of-the-art research codes to exploit advanced computer architectures involving both vector and parallel processing concepts, (2) developing visual techniques for analyzing computational results and data acquired from measurement or sensing equipment. (3) developing advanced data communications techniques and capabilities for use in local-area as well as wide-area networks with scientific and engineering computing applications, and (4) developing programs that result in tha transfer of advanced computing and telecommunications hardware and software technology to the university and private sectors. There is particular 
emphasis on Grand Challenges in areas in which Argonr $:$ scientists are preeminent. (See also ANL-1, 2, and 8.)

\section{National Research and Education \\ Network (NREN)}

Inseragency Inserim NREN See ANL-10.

Basic Research and Human

Resources

Basic Research

See ANL-1,4,5, and 31 .

Research Participation

ANL-47 Since 1987, Argonne's Mathematics and Computer Science Division has and Training held an annual Summer Institute in parallel programming. The objective is to enable graduate students and postdoctoral researchers to leam about advances in parallel computing and to experiment with new ideas on state-of-the-art machines. The agenda includes half-day lectures from invited speakers and practice in developing parallel programs on the ACRF machines. The institutes are sponsored joindly by DOE and NSF. Also, for the past several years, this division has conducted bimonthly classes in parallel computing. The participants include researchets from universities, industry, and various research laboratories throughout the world. The classes focus on the ACRF environment, offer ample hands-on experience, and help the attendees apply parallel programming to their own research areas. See also ANL 5 and 46.

Infrastructure

Education, Training, and Curriculum
See ANL-1, 2, 3, 5, and 10 .

ANL-7 Education has become an increasingly important responsibility of those involved in high-performance computing. Argonne is coordinating a new projech, called A-PRIME (Argonne PRogram In Mathematics Education), aimed at strengthening teachers' understanding of the principles of mathematics. The project involves establishing a Math Visualization Laboratory at Chicago-area universities to train future and practicing teachers in mathematics. Other activities include special courses in supercomputing offered to minority college students and a minority internship in parallel computing for a faculty member from a recognized U.S. institution. (See also ANL-47.)

\section{Computing and Telecommunications Technologies}

Advanced Computing Systems

Vector Supercompusers

ANL-8
The Argonne Cray X-MP/18 is a high-performance, single-processor computer with a CPU cycle time of 8.5 nanoseconds and a theoretical maximum result tate of 235 million floating-point operations per second for concurrent multiply-and-add instructions. It has eight million words of memory, with up to seven million words available to a single user program, and it provides gather-scatter instructions, which improve the performance of applications relying on the indirect addressing of array elements. The Cray X-MP has over 20 gigabytes of directly attached disk storage and has file-transfer access to over 25 gigabytes of public user disk storage on the central IBM and central VAX cluster computers. 
The Argonne Cray can access six directly attached IBM 1600/6250-bpi nine-track tape reel drives and four 38,000 bpi cape cartridge drives, all of which are shared with the central IBM systems. The major operating system components of the Argonne Cray are UNICOS, the Networking Queuing System, the X Window System, and the Network File System. Software available includes programming languages, the Disspla graphics librax $y$ (with an $X$ Window interface, mathematical subroutine libraries, Cray-supplied UNICOS software libraries of mathematical functions, routines for Fortran-specific features, L/O routines, scientific application routines, and utilities, and the Cray-supplied UPDATE source code maintenance utility. Argonne researchers also access Cray Y-MP and Cray-2 multi-processor computers at the National Energy Research Supercomputer Center, at the Cray facilities at Mendota Heights, and at various other sites through either federal or industrial grants or through reciprocity agreement. (See also ANL -4 and 5.)

Parallel Processing

Diskribused Computing

Compilers/Prngramming Systems

Compiler Performance

Data Communications

Graphics Networks

Teleconferencing

Migh Speed Networking
See ANL-1, 23 , and 4.

See ANL-10 and 36 .
ANL-9 Argonne researchers are assessing the viability of the emerging Fortran 90 language standard as a technology for high-petformance scientific and engineering software development. Because the overwhelming majority of technical software in use today is written in Fortran, early efforts have involved case studies of code conversion from Fortran 77 to Fortran 90. Related work has included determination of structures, tools, and methods appropriate for development and maintenance of Fortran 90 mathematical software libraries, and assessment of the commercial viability of the language. Ongoing research is addressing the use of Fortran 90 as a means of facilitating effective and scalable adaptation of existing codes to parallel computing environments.
See ANL-10.

See ANL-10.

ANL-10 Argonne provides computer users with a variety of communications capabilities, including both onsite and remote access to local, Laboratory-wide, national, and international networks. These capabilities involve a wide range of telecommunications technologies, from simple point-to-point data communication links to sophisticated networks with complex software and hardware configurations (e.g., network bridges, gateways, routers, and servers). Argonne networks include the Transmission Control Protocol/Internet Protocol (TCP/P), the Digital Equipment Corporation network (DECnet), AppleTalk, and the Xerox Network Sysiem (XNS) protocols. Network software and hardware allow users to transfer files, provide interactive access, send electronic mail, and run application programs on computing systems connected to networks. Through the Laboratory-wide NJE network. users of distributed computers can access the Argonne central computers, along with associated resources and services. 
Data Management and Storage

Database Management

\section{Visualization and Imaging}

Scientific Visualization

Inseractive Visualization
The current Argonne network lopology includes local area networks based on Ethernets encompassing buildings or programs, a Laboratory-wide network currently based on an Ethernet-compatible capability (LANmark), and gateways to national and international networks (ESnet, HEPnet, MFEnet, CICNet, NSFnet, BITnet, and Comett). Most of these networks, including ESnel, are hased on high-speed $\mathrm{T} 1$ links with 1,544 megabits-per-second technology. Within the next several years, many of these networks will migrate to T3 with 45 megabits-per-second technology and to fiber-distributed data interfaces (FDDI) with 100 megabits-per-second fiber-optic rings. .. gonne has identified FDDI networking as an important development in allowing researchers to take advantage of high-speed, liber-optic networking.

See ANL-6.

ANL-6 Argonne researchers are developing object-oriented database strategies that will meet the next-generation needs of the high-energy physics community. Foremost among their concerns is that the amount of data to be generated by detectors at the Superconducting Super Collider will test the limits of storage and access technology. While only a fraction of the information generated will be saved, that fraction alone may require up to 1,000$)$ terabytes per year of storage.

ANL-11 Argonne researchers use visual analysis of collected data and computational results on machines ranging from supercomputers (c.g., the Argonne Cray X-MP) and supergraphics workstations (e.g., the Sparcstation. Personal Iris, and Macintosh II). Argonne computer scientists are continually developing advanced scientific visualization lechniques ranging from new algorithms to high-speed networking in collaboration with their Argonne colleagues to meet application-specific needs. Visualizations of molecular dynamics, huid dynamics, structural mechanics, volumes, weather cycles, computer code pertormance, and other subjects are now under way. (See also ANL-12.)

ANL-12 Computational results from simulations run on the Cray X-MP may be pustprocessed on the Stardent Titan to produce sophisticated three-dimensional animations. A sizeable community also uses the Disspla graphics library for production computing on the Cray, VAX. and IBM computers. Argonne has extended the capabilities of computer codes using the Disspla graphics library by providing local extensions to the $\mathrm{X}$ Window system Disspla driver. These extensions allow users of $\mathrm{X}$ Window workstations to interact with Disspla graphics programs running on the Cray or VAX to capture animations for later viewing on workstations and to produce video recordings. Applications such as NCS A Image and NCSA DataSlice (for Macintosh, Vaxstation, or Sparcstation workstations) also provide substantial capabilities for data analysis. Convenient video services are available to users who create animations on distributed and central computers. A Lyon Lamb RTC scan convertor accepts images from a wide variety of workstation platforms. An Abekas A60 digital framestore enables frame accurate 
animation recording. Available video formats include VHS, 3/4 Umatic, and Betacam broadcast quality cassettes. (See also ANL-11.)

Image Archiving Systems

Distributed Visualization
See ANL-12.

See ANL- 11 and 12.

\section{Computational Science and Engineering Applications}

\section{Biology and Life Sciences}

Compusational Biology

Radiation Biology

Structural Biology

Genome Research

\section{Chemical Engineering}

Multi-Phase Flow
ANL-13 Argonne is investigating the use of logic programming techniques and automated reasoning for the study of RNA. We have developed a logic programming toolkit that has been used to design a tailored computer search of the secondary structure of an RNA molecule. We are also investigating the use of logic programming to find the most reliable signature positions of the ribosome in coli. ("Signatures" are features within the ribosomal sequence that characterize certain groupings or phylogenetic subgroups of bacteria.) In the area of high-performance computing, Argonne is studying the potential of parallel processing for large-scale numerical computations in molecular biology. To date, this work has led to the design and validation of a parallel algorithm that successfully aligns RNA sequences.

ANL-14 Argonne's radiation biology programs integrate results from a broad spectrum of studies, with the goals of clarifying the basic processes in cancer induction and developing unifying concepts of radiation damage. Chemical studies examine events that occur at the cellular and molecular levels during processes such as tumor development, cell differentiation. genetic change, and cell response to toxicants. With the objective of gaining additional insight into the complex radiation effects in biology, Argonne researchers are also studying the underlying physics and chemistry in interactions of ionizing radiation with matter. The current research program involves two main areas: (1) determining and compiling accurate cross-section data of electron-atoms/molecules/surfaces and ion-atoms/molecules/surfaces collisions and (2) developing and refining electron/ion transport theory, which is the fundamental theory for describing electron/ion behavior in terms of space and energy in matter.

ANL-29 Research in structural biology seeks to determine the structure of macromolecules, as a step toward understanding the relationship between the form and function of molecules in living matter. In biotechology, an emerging research area at Argonne, studies of photosynthetic reaction centers are under way. (See also ANL-14.)

ANL-15 Argonne genome research ranges in scope from epidemiological studies of human populations to studies of biological processes at the whole-animal, cellular, and molecular levels. Such work employs a variety of research techniques, including recombinant DNA technology, cell culturc, and protcin crystallography. 
Slurry (Coal) Systems

\section{Chemistry}

Fundamental Investigations

Combustion Processes

Molecular Dynamics/Atomic, Molecular, and

Electronic Structure
See ANL-39.

ANL-17 Research in chemistry encompasses a broad spectrum of fundamental investigations of atomic and molecular phenomena and chemical reactions. The research is concerned with central issues in the fields of radiation and photochemistry, natural and artificial photosynthesis, basic electron transfer processes, theoretical and computational chemistry, the chemical physics of elementary reactions, transition metal cluster chemistry, coal structure and coal conversion and separation science. Supporting these activities are advanced instruments, including a picosecond electron linac, a high-intensity tunable vacuum-ultraviolet light source, time-domain magnetic resonance spectrometers with fast optical detection, a novel cluster chemistry apparatus, high-field solid and solution nuclear magnetic resonance spectrometers, very high resolution mass spectroscopic equipment, and an array of computational equipment ranging to mini-supercomputers.

ANL-18

Attaining significant improvements in combustion efficiencies requires understanding the interplay among the various substances involved and the quantum chemistry that causes those substances to react. Studies involving computer simulations of gaseous and solid fuel combustion are under way at Argonne to understand better how to increase tnergy along with efficiency, achieve more complete burning, and produce by-products more acceptable to the environment. Simulations of gaseous fuel combustion allow detailed analyses of fluid flows and reactions. Simulations of solid fuel combustion assist in clarifying the synthesis of advanced metallic and ceramic materials for high-temperature engine components. Available computational technology now allows computerized modeling of combustion phenomena involving highly nonlinear time-dependent systems of partial differential equations. As researchers vary critical parameters of the problems, they obtain new solutions through a sequence of bifurcations. An adaptive pseudo-spectral miethod enables them to describe numerically the solutions on bifurcation branches well beyond the bifurcation point (the region where analytical methods work well). In particular, they have obtained both steady-state solutions with a fixed number of cells and regions of bistability in the parameter space where two different cellular solutions can occur stably. Further, they have found standing wave solutions--solutions that exhibit pulsations in time as well as cellular spatial structure.

ANL research in chemistry encompasses a broad spectrum of fundamental investigations of atomic and molecular phenomena and chemical reactions. The research is concemed with central issues in the fields of radiation and photochemistry, natural and articifical photosynthesis, basic electron transfer processes, theoretical and computational chemistry, the chemical physics of elementary reactions, transition metal cluster chemistry, coal structure and coal conversion, and separation science. Theoretical studies in the Cluster Chemistry Group are focusing on the structural and dynamic properties of atomic clustets and on their interactions with molecules. The Theoretical Chemistry Group is studying the chemical dynamics of eleinentary reactions, primarily in combustion. To examine problems in 
environmental restoration, catalysis, and coal formation, the Coal Chemistry Group is modeling the interaction of organic molecules on clay surfaces.

\section{Condensed-Matter Physics}

Crystal Growth

NU.49

Energy S'ystems

Systems Analysis and

Simulations

Environmental Sciences

Global Climate Modeling
ANL,-21
Crystals have long been known to be composed of pariodically repeating packing, units. Since it is impossible to make a periodic structure with fivefold symmetry, crystals with fivefold symmetry have been assumed to be impossible. The discovery of solids with fivefold symmetry--but with crystalline diffraction patterns--has provided a puzzle to physicists interested in the structure of materials. The Materials Science Division, in collaboration with Northwestem University, has been simulating various models for these "quasicrystals." The properties of these models ase then compared with the measured properties of quasicrystalline materials, to understand better the structure of this intriguing now state of matter. These models suggest that the quasicrystal structure is stabilized by the high entropy available because of the large number of possible arrangements, rather than by special interactions that make the quasicrystal structure the structure of lowest energy. Experiments to distinguish among the various mdels are in progress in many places, including Argonne's Intense Pulsed Neutron Source.

(NL-20 Argonne scientists are deveioping and refining a high-performance simulation environment for the analysis of advanced energy systems concepts such as fission, fusion, fossil, MHD fuel cell, and geothermal. This environment includes parallel/distributed computing, object-oriented software environments, and knowledge-based information systems, with the common goal of aiding the development and mainteriance of high-performance simulation systems that exploit state-of-the-art computers.

In attempting to predict long-range weather and climate variations with greater accuracy, Argonne computational scientists are analyzing data reflecting the many ways in which the atmosphere, the oceans, and the biospheric conditions combine to cause these variations. More specifically, these scientists are attempting to understand better the carbon dioxide atmospheric dynamics that may govem perturbations resulting from man-made releases of chemicals and energy into the atmosphere, the oceans, and the biosphere. Currently, these scientists are performing a multi-year run of the National Center for Atmospheric Research Community Climate Model to understand better the effects of increased carbon dioxide, a so-called "greenhouse gas," on the global climate. This Model simulates the movemeril of the atmosphere on a global scale as well as the physics of solar radiation, cloud cover, and other physical systems. The project, which entails a simulation of up to 30) years, is being conducted on the Cray X-MP. In a related effiurt aimed at developing a new-generation global climate model (which would run with many hundreds of processors working in parallel), Argonne researchers are identifying and characterizing patterns of processor load as the existing Model executes over the grid squares that represent the atmosphere. 
Atmospheric Sciences

Earth Sciences

Ocean Circulation

Simulation

High Energy Physics

Quantum Chromodynamics
Argonne is also collaborating with the National Center for Atmospheric Research and with ()ak Ridge National Laboratory in DOE's CHAMMP (Computer Hardware, Advanced Mathematics, and Model Physics) Modeling Program in support of parallel climate modeling. Efforts focus on four areas: scalability analysis, parallel algorithms and numerical methods, tools, and load distribution. We have, for example, completed a comprehensive analysis of the parallel scalability of the spectral transform method, widely used in climate models, and have constructed prototype implementations of this and several other methods. We ure also developing a graphics-based user interface for the advanced climate model, and have begun work on techniques for distributing physics computations to the nodes of a parallel computer in order to correct load imbalances. (See also ANL-22.)

See ANL-21 and 22 .

ANL-22 Environmental systems analysis is a relatively new area of research involving the application of computationally intense procedures to oceanogruphic, atmospheric, and terrestrial systems. Research includes studies of sampling networks (necessary to establish fundamental design criteria for sampling spatially nonhomogeneous variables in natural systems), of model uncertainty (necessary for establishing realistic confidence limits on model projectionss), and of inverse lechniques iwhich offer an innovative approach to the identufication and parameterization of environmental processes). One computational detivity involves the development of nonparametic statistical methods based on Monte Carlo resampling to determine the expected accuracy and precision of environmental sampling networks as a function of network size. These methods will include methods of combinatorial optimization such as simulated annealing to the problem of selecting an optimal subset of sampling locations for application to environmental surveys and will involve the design of efficient algorithms for sequential and parallel implementations.

See ANL-22.

ANL-23 Theoretical rescarch in high energy physics encompasses quantum chromodynamics and the application of advanced computing techniques to lattice gauge theory. The resulting simulations of the dynamic behavior of mixat quantum-classical particles involve using a split-operator technique (to handle the time-evolution operator for the electron wavefunction) to accommodate the kinetic energy in momentum space and the potential energy in real space, while using ihree-dimensional Fourier transforms to make the transformation between real and momentum space. This technique is the only one currently that can reveal the dynamic behavior of the quantum particle as well as the behavior of the classical system. One Argonne project involves (1) computing masses of strongly interacting particles (hadrons) from fundamental quantum chromodynamics, (2) studying the thermodynamics of quanturn chromodynamics, particularly the chiral phase transition of ruclear matter at high temperatures, and (3) studying the strong coupling phase transition of quantum electrodynamics and determining the properties of the chiral symmetry broken phase. 
Detector Design

Accelerator Design and Simulation

Data Acquisition and Analysis

\section{Materials Science}

Electronic Structure

Neutron-Scaltering Research

ANL-30

Bulk Solids and Surfaces

ANL-24
Argenne researchers are using Superconducting Super Collider detector simulation programs to provide critical evaluations of the potential performance of the proposed detector. Currently, they are reconstructing data from large detectors to determine properties of particles produced in a collision. The resulting datasets will be scanned repeatedly with inodest processing to produce graphics displays of physics quantities. (See also ANL-25.)

ANL.25 Argonne carries out both experimental and theoretical research in partiole physics. Much of this research is concerned with the development of large-scale detector facilities constructed and used in collaboration with university physicists, at particle accelerators located elsewhere, notably at Fermilab and at the HERA i-p collider being built in Hamburg, Germany. This accelerator-based program is focused on searches for new particles and particle states, as well as upon studies of quarks and gluons and their fragrnentation into jets of hadrons.

Advanced accelerator research and development concentrates on the use of wakefields for high gradient acceleration. In oher major experiements, conducted in an underground laboratory in the Soudan Iron Mine in Minnesota, Argonne searches for proton decay and stable-matter quarks.

See ANL-24.

ANL-26 Under study at ANL are the electronic properties and behavior of advanced matcrials important to science and technology. Research in this area ranges from fundamental investigations at the atomic level to studies of the macroscopic behavior of materials in harsh environments. Currently, ANL is developing alternative approaches to improve the capability to deal with the electronic structure of larger systems, including parallelization, advanced optimization algorithms, advanced eigensystem upproaches, basis set modifications, and altcr native formulations of density functional theory such as path-integral methods.

Involving in part the study of electronic structure, the Intense Pulsed Neutron Source (IPNS) is a national user facility for neutron-scattering research. Research is under way in IPNS to examine the structure of such materials as high-'emperature supercondictors, alloys, composites, polymers, the myelin sheath that insulates nene cells, catalysts used in oil refining, and materials for advanced energy teshnologies. Recently developed instruments are being used to study properties near the surfaces of magnetic and polymeric materials and the structure of liquid and noncrystalline materials. High priority is placed on making the facility and its instruments as easy and as convenient as possible for visiting scientists to use through ancillary equipment and computer facilities. The full-time activities of eight to ten scientific assistants and associates help users in carrying out experiments and in collecting and analyzing data. The ten neutron scallering instruments produce large quantities of data that must be analyzed in a timely manner.

ANL-27 ANL research into the basic physics of surface and interfacial phenomena examines processes such as oxidation, corrosion, catalysis, 
and cohesion; produoes materials with tallored surfaces; and develops new techniques for surface analysis. Through fundamental investigations relying heavily on the Eleotron Mioroscopy Center, ANL explores the effects of point defects, aggregates, and precipates on the properties of the solide in which they form during irradiation. (See also ANL-26.)

Superconductivity

Mathematics and Numerical

Methods

Algorithmic Research

Linear Algebra
ANL-28

A broad multidisciplinary effort is devoted to the study of oxide materials having high superconducting transition temperatures. For example, to understand better the structure und interactions of vorticus in layered superconductors in a mixed state. ANL has adoptind the tectmique of numerical mindmization of the Cinzatitg-Landepu free energy function and the Monie Carlo simulation of the voriex pliate diagram. Because of interest in the collective bahavior of iarge numbers of such vortices, the problem is computationally intensive, taquiring the use of parallel algorithms or hybrid parallel and supercomputer techniques. (See also ANL-26.)
ANL.31

Research in mathematics and computer science is focused on the development of algorithms and software, the analysis and application of mathematical methods for the solution of scientific problems, and the development of computer science methodology and tools to advance the state of the art in scientific computing. Activities range from theoretical research on automated reasoning to the analysis of nonlinear phenomena.-for example, in combustion, fluid dynamics, and diffusion processes--to research in automated reasoning and abstract programming. Argonne's many new advanced-computing efforts include, for example, logic programming and multiprocessing for molecular biology and scalability analysis of parallel algorithms for climate modeling. Much of the research focuses on parallel architectures and is supported by the ACRF. Argonne makes a special effort to collaborate in advanced-computing research with scientists from universities, other nutional laboratories, and industry.

ANL-43 In numerical linear algebra, efforts focus on the development of algorithms and software for solving large sparse-matrix problems on parallel machines. In particular, we are developing efficient software for orthogonal decompositions and variants of the conjugate gradient and Lanczos algorithms. The goal is to produce algorithms and software that are well suited for large-scale parallel environments and that can be used to solve numerically difficult problems rellably (as well as numerically casy problems with great speed). These characteristics are crucial for the study of ill-posed problems that arise in many applications (c.g. reservoir modeling and atmospheric temperature profiles). A major achievement has been the development of the LANZ software package for solving the large, sparse, symmetric generalized eigenproblem on vector stupercomputers and shared-memory parallel computers. This problem is of significant practical importance in, for example, struc' Iral engineering where it arises in the vibration and buckling problems, Our efforts in numerical linear algebra also involve development of efficient. yet portable algorithms for the solution of linear equations. The 
Approximation Techniques

ANi. 32

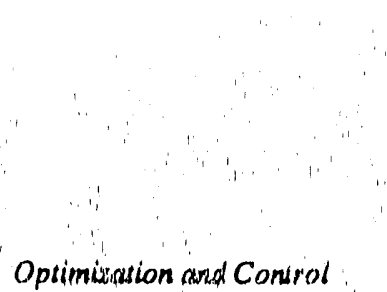

Optimiration and Conirol

Differential Equations

Nom-Numerical Methods algorithms dovelnped are being incorporntod in a new software package, L.APACK, whioh we expect to become the staridard software for linear ulgebra on advances corroputers. (See also ANL.31.)

Ou: reseatylit in function approximation techricfues seetis to improve the quality of the pristitive, elementas $y_{t}$ and spicial functions ensential to scientific computing and to advarice the use of function approximation techniques in cther atdus of scientific computing. We are developing accutrath sevit mothods and upplying them to a large olass of elementary functions. Our work ori the power funotion, for example, led to a new aigurithm that computes $x^{4}$ to full machino accurscy; this algorithm was adoptsal by Sun Microsystems and MIPS Computer Inc. (Spe also ANti, (1)

ANL-33 Our resentichenters on the devisupment of algorithms ast software for thit solution of large. scals nonlinear op itrization problems. Of fartccila: interest are, the optimization problems that arise in geophysical oil prosperting, modeling of inhomogeneous superconducting materials, and chemilital kinetics. For example, we are developing now computational methods for seismic prospecting that will enable scientists to model complex geological regions, while keeping the number of parameters in the model small enough that the inversion problem is tractable. We have also begun work on the design of parallel algorithms for discrete-time uptimal control problems. Finally, as an extension of our work on the MINPACK software package, we are collaborating with the Army High Performance Computing Research Center at the University of Minnesota to produce algorithms and optimization software for high-performance computers. This effort includes developing a collection of significant test problems from real applications in such diverse fieids as fluid dynamics, medicine, combustion, lubrication, and superconductivity. (See also ANL-31.)

ANL-34 Many scientific problems -. for example, in fluid dymamics and combustion--give rise to partial differential equations (PDEs). Our work focuses in particular on methods for solving the system of linear equations that arise from implicit differencing methods (either finite differences or finite elements) applied to PDEs in two and three spatial dimensions. We are investigating domain decomposition methods-numerical methods that are based on dividing the physical (or computational) domain up into many subproblems. Such a decomposition provides a natural method for parallelizing the solution of a PDE. Our techniques appear promising: problems with hundreds of thousands (in two dimensions) or millions (in three dimensions) of unknowns have been solved on a variety of parallel machines, including a BBN TC2O(O) and an iPSC/860. (See also ANL.-31.)

ANL-35 The objective of the research in automated reasoning is to produce a program that can function at the level of a colleague. Our current program, OTTER, provides a laboratory for studying new strategies and testing their value on various difficult problemss in areas such as circuit design, program verification, and theorem proving. We have also developed a parallel automated reasoning system called $R(O)$, and are investigating its use on shared-memory and distributed-memory computers. (See also ANL.31.) 
Programming Environments and Performance Analysis

\section{Mechanical Engineering}

Compusational Fluid

Dynamics/Mechanics

Heat Transfer

Material Forming

Modeling.-Casting

Slurry Modeling
ANL-36

As the potential utility of parallel processing becomes increasingly apparent, the need for a software technology that allow: in straightforward use of the next generation of parallel machines also increases. The focus of our research is the development of a software technology that allows effective use of highly parallel multiple-instruction multiple-data (ivilMD) systems. Among tho tools currently being developed are (1) a portable sel of cornmurtication primitives, $\mathrm{p4}$, that provides for efficient portable programming of shared-memory machines; (2) a portable implementation of the high-level language PCN, which simplifies dovelopment and maintenance of complex programs and provides interfaces to Fortan and $C$ to nllow reuse of existing code; (3) a graphical performanre visualization tool, Atrace, which produces an animated and a statistical display of a program's logfiles, (See also ANL-31.)

ANL-37 A major area of investigation in the design, development, and safety evaluation of nuclear reactors and other energy systems is the thermal hydraulic behavior of a variety of complex systems under diverse operating conditions. As a result Argonne has developed a series of general purpose computer codes for the analysis of three-dimensional fluid transient problems. As this effort has grown and matured, many versions of these codes have evolved into tools applicable to inciustrial and technical areas outside the field of reactor development. For example, Argonne researchers are developing a fast production-level computer simulation program capable of addressing design, scale-up, erosion/corrosion and combustion complexities in fluidized beds in a realistic time scale and in an economical manner to minimize the time associated with costly pilot-scale work. This project would benefit industries that use Illinois high-sulfur coal and research institutions that promote high-sulfur coal. The project would produce a high-performance version of the FLUFIX (1) computer program tailored for multiprocessor shared-memory engineering workstations and would develop user-friendly software packages for input data automation and computer-generated output data visualization. As a result of this project, state-of-the-art multiphase computational fluid dyriamics computer models ard solutions could be made available to Illinois industries, in particular throu'gh a high technology resource center and ongoing workshops for Illinois fluidized-bed combustor boiler manufacturers and operators. Whilc reactor-related problems remain a major concern of this effort, a varicty of other computationally intense areas are now receiving attention. (Sce also ANL.-41 and 42.)

See ANL-37,

ANL-38 Argonne scientists are developing numerical methods to model the mold-filling and soliclification process used in typical foundry operations. One goal is to interact with industry to provide a computer code that can be used to moael casting operations, that is user-friendly, that runs on a small workstation, and that can be operated by a person without special knowledge of thermal hydraulics or computer systems.

ANL-39 Another activity is the modeling of slurries. Reseachers are developing analytical models, based on first principles, for dense slurry flow in 
common geometries relevant to transporting fossil fuels. A further step is to develop an engineering/computational system that uses these models to provide design information to the fossil industry without having to preform extensive application-specific experiments.

Nuclear Engineering

Reactor Design

Reactor Safery and

Transient Analysis
ANL -40

Argonne specializes in advanced liquid metal and breeder system nuclear reactor design and analysis but is also acive in design and analysis of diverse research and isolope production reactors. The analysese focus on safety, economics, efficiency, and waste minimization. One design project is the modeling of laminated elastomer bearings, where computational activities include the development of algorithms (1) to model three-dimensional static and dynamic responses of seismic isolation bearings, and (2) to simulate the hysteretic response of elastomers. Visualization capabilities are under development to display the motion and numerical results of isolation bearings through dynamic simulations. (See also ANL-37 and 41.)

ANL-41 Reactor safety studies at ANL piovide insights into preventing reactor accidents and mitigating their potential consequences. Safety analysis projects under way include computer modeling of (1) missile impacts against reinforced concrete and prestressed concrete structures and (2) responses of reactor structures under earthquake loads.

Cumputer simulations being developed include the responses of piping loops subjected to dynamic loads and the responses of reactor vessel and in-vessel components to severe accident transients. These simulations include fluid-structure interactions involving reactor vessel and various other component structures under severe accident loads. Reactor system transient three-dimensional thermal-hydraulic behavior is analyzed with a version of COMMIX that combines algorithm improvements, code optimization, and model enhancements. These changes drastic ally improve the performance and capabilities of COMMIX for advanced and commercial reactor analysis. Argonne is also developing algorithms for simulating reactor primary systems subjected to reactor transients with neutronic behavior coupled to thermal-hydraulic phenomena that vary across the reactor. Some of these simulations are interactive three-dimensional animations. (See also ANL-37.)

Argonne Fresendly performs reactor physics analysis usizg whole-core three-dimensional nodal diffusion and transport calculations. These calculations are applied to many problems, including (1) the determination of reactor feedback coefficients and their effects on transient and safety performance and (2) depletion simulations used to minimize high-level waste, optimize fuel cycle economics, or minimize control rod requirements. The cross sections used are produced by the collision probability integral transport method, incorporating uniquely complete spectral detail. In the resonance ranges, such detail can be crucial to the fidelity of the analyses. Work on other methods will provide (1) a full-core three-dimensional discrete ordinates transpon capable to account better for large-scale heterogeneities and (2) a subassembly transport capability to produce subassembly or pin-cell cross sections for complicated heterogeneous subassembly designs (e.g.,

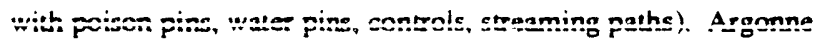
scientists will also conduct whole-core, three-dimensional, five-group neutron transport calculations suitable for depletion and kinetic analyses. 
Fusion Systems

Online Reactor Diagnostics

ANL-45

See ANL 41 .

Siructural Analysis

ANL-42

\section{Structural Engineering}

\author{
Analysis \\ Earthquake Analysis \\ and Response \\ Fatigue and Failure
}

onne scientists are developing algorithms for three-imensiona analysis of liquid metal magnetohydrodynamic (MHD) flows in electrically conducting and nonconducting ducts and manifolds of $v$ arious geometries in the presence of strong magnetic fields. The geometries considered are representauve of those typically encountered in the design of fusion reactor blanket modules.

Argonne is developing statistical techniques for monitoring the inherent core feedback processes in advanced liquid metal reactors. These feedback processes are purposely engineered at the plant design stage to ensure a safe reactor response to various reactor accidents. The reliability of obtaining a safe response is improved further if these processes are then monitored online in the as-built plant and compared with values deemed safe. In a commercial plant, this comparison must be done in real time, on the order of minutes. Presently, however, the results of the comparison lag behind the data collection window by several hours, because of the intensive computational demands imposed by the statistical techniques. The use of parallel processing is one avenue being pursued to shoiten this delay time.
Detailed, exact Monte Carlo neutron transport simulations are used at to provide benchmar! solutions for approximate transport methods. Argonne is developing suitable algorithms to enable use of Monte Cario methods for simulating neutron transpon in nuclear reactors on higinly parallel supercomputers. Such algorithms will make currently untenable (because of computer limitations) Grand Challenge applications tenable, such as depletion analyses for fuel and bumable poisons in commercial reactors.

See ANL -40 and 41.

ANL-44 Finite element analysis is the dominant tool in the numerical solution of the partial differential equations governing the nonlinear transient analysis of structural or continuum problems and in a variety of other areas as well. Finite element codes possess considerable versatility in modeling complex shapes and boundary conditions. The WHAMS-3D computer program employs explicit time integration to do nonlinear. transient analysis of frames, shells, plates, and continua in three dimensions. This program has been developed jointly at Northwestern and at Argonne and is intemationally recognized as a state-of-the-art program for performing nonlinear transient analysis. WHAMS-3D has maintained its role as a leading-edge program through extensive research in innovative methods for enhancing computational efficiency.

Order-of-magnitnude increases in the computational speed over a vector supercomputer have been attained in research conducted at Argonne for three-dimmsinnal nonlinear analysis of sholl smirnures on SIMn architectures. This speedup was difficult to obtain, and substantial 
redesign of the algorithm and underlying data structure was necessary and incorporated in an "Exchange" algorithm that allowed minimization of interprocessor communication at the expense of redundant computations and storage. A combination of the Exchange algorithm and the Argonne-developed $p 4$ primitives has resulted in portable dynamic finite-element programs. Once the algorithm is restructured as a set of processes communicating through messages, the program can run on systems as diverse as a uniprocessor workstation, multiprocessors with or without shared memory, a group of workstations that communicate over a local ares network, or any combination of the above.

Argonne has employed this program to perform analysis of various reactor components and structures. Dether organizations have employed the program to perform analysis of ice forces on Arctic structures. analysis of buried structures, military weapon analysis, aircraft engine structural analysis, analysis of electronic components, reactor safety fluid-structure analysis, impact and penetration analysis, and automobile crashworthiness simulations. The program has been extensively validated by comparisons with a wide range of experiments associated with large deformations, buckling, impact-penetration, etc. (See also ANL.40.)

In studies of crashworthiness, impach, and penetration, it is not unusual for an analysis to require 100 hours of CPU time on current production supercomputers despite the simplicity of the models being studied. It is difficult to obtain blocks of time this large in a production environment. Weeks often pass before the results of a single simulation are obtained. Coupled with the iterative nature of the design process, the simulation task may stretch into months. Consequently, the role of such simulations in engineering decisions is impaired and the scope of design options severely restructed to meet industrial time schedules. Attention has focused on high-performance computit architectures as effective avenues for bridging the gap between computational needs and the power of computational hardware. With these powerful tools at their disposal, engineers would be able to tackle sophisticated simulations that are currently intractable without the use of empirical data and the separate modeling of each region of the problem to break the problem up into models that are manageable. Nonlinear dynamic finite-element analysis with explicit time integration is employed to model these phenomena. Computational scientists in the Argonne Computing and Telecommunications Division are studying the efficient application of high-performance computer architectures to address finite-element simulations. (See also ANL-44.)

\section{Technology Transfer}

Industrial Partners in Compusing
ANL-46

Argonne's Advanced Computing Research Facility has an active affiliates program for both universities and industrial organizations interested in using the ACRF multiprocessing systems. The Acarlemic Affiliates Program currently includes 28 colleges and universities, the Industrial Affiliates Program 15 members. (See also ANL-31.) 
Industrial Partners in

Science and Engineering

Other software developed at

Education. Training, and Human Resources
ANL-48 Argonne transfers technology to industry in a variety of ways, including cooperative research and development, contract research, staff exchange, and licensing of inventions. Argonne seeks early industrial involvement and follow-through with necessary adaptation and training as new technology moves to the shop floor. As a multi-disciplinary laboratory, Argonne has the full range of scientific and engineering skills needed to use leading-edge computing capabilities. Argonne has developed extensive links with industry and with economic develanment organizations at the state, regional, and local levels. These ties, combined with relationships with universities and colleges, make Argonne a major resource for the industrial community. In one such interaction, the Advanced Software Development and Commercialization Project, Argonne scientists worked with CSRD and a variety of industrial firms (notably General Motors and Inland Steel) to apply software programs to structural problems in sheet metal. The WHAMS computer program, originally developed by Argonne and Northwestern University was used to perform the analyses.

Argonne, CSRD, and local firms such as the Nunierical Algorithm Group (NAG) were used to enihance the code to run on high-performance vector computers, massively parallel computers, and scientific workstations.

See ANL-31 and 46 

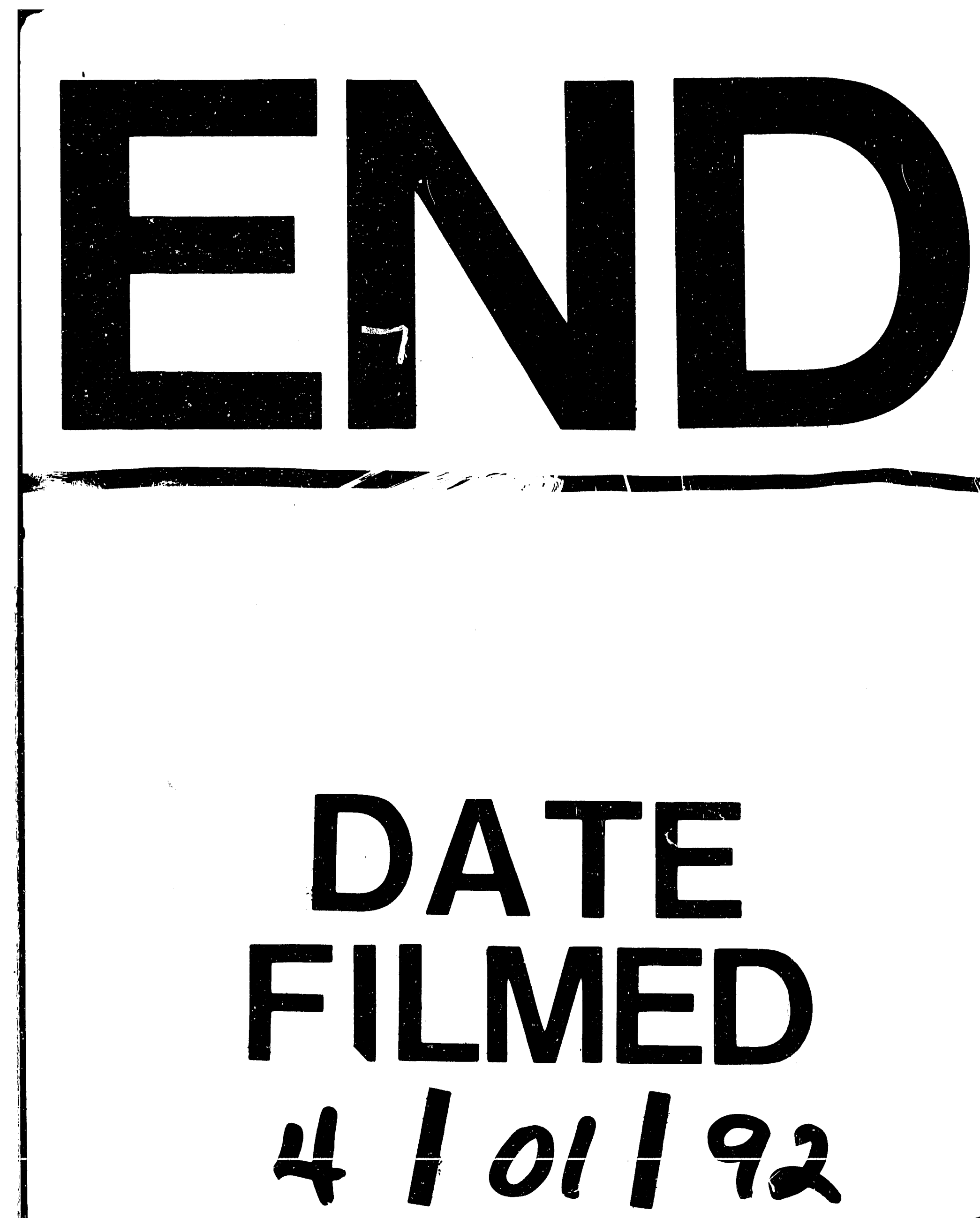

$I$. 
